\title{
Fundamental Immunology of Skin Transplantation and Key Strategies for Tolerance Induction
}

\author{
Junyi Zhou $\cdot$ Weifeng He $\cdot$ Gaoxing Luo $•$ \\ Jun Wu
}

Received: 17 October 2012/ Accepted: 26 April 2013/Published online: 18 May 2013

(c) L. Hirszfeld Institute of Immunology and Experimental Therapy, Wroclaw, Poland 2013

\begin{abstract}
Transplantation of allogeneic or xenogeneic skin grafts can evoke strong immune responses that lead to acute rejection of the graft tissues. In this process, donor-derived dendritic cells play crucial roles in the triggering of such immune responses. Both the innate and acquired host immune systems participate in graft rejection. At present, the rejection of skin grafts cannot be well-controlled by ordinary systemic immunosuppression therapy. Although several strategies for the long-term survival of allogeneic or xenogeneic skin grafts have been demonstrated in animal models, the induction of long-term tolerance to skin grafts is still a great challenge in clinical settings. In this article, we review the progress in the understanding of immune responses to skin grafts and discuss the possible methods that can decrease the immunogenicity of graft tissues and improve the survival of skin grafts, especially those included in preoperative pre-treatments.
\end{abstract}

Keywords Skin transplantation - Immunology

\section{Introduction}

The successful transplantation of allogeneic organs and tissues has significantly improved the outcomes for patients with severe organ dysfunctional diseases and is one of the greatest surgical and immunological achievements of the twentieth century.

J. Zhou · W. He · G. Luo · J. Wu ( $₫)$

State Key Laboratory of Trauma, Burn and Combined Injury, Institute of Burn Research, Southwest Hospital, The Third

Military Medical University, Chongqing, China

e-mail: junwupro1@yahoo.com.cn

W. He $\cdot$ J. Wu

Chongqing Key Laboratory for Proteomics of Diseases,

Chongqing 400038, China
The transplantation of allogeneic skin grafts is an important therapy for patients with major burns or chronic refractory wounds. However, allogeneic skin grafts evoke potent immune responses. Following the transplantation of skin onto a healthy MHC-mismatched recipient, a quick immune rejection process will lead to the failure of the graft within 10-14 days (Richters et al. 2005) with characteristic skin tissue destruction. Allogeneic skin grafts are rejected in a short time period of approximately 20-30 days, even if grafts are placed over the fully excised burn wound base (Bravo et al. 2000; Hansbrough et al. 1997). This process could hardly be controlled by currently available immunosuppressive agents (Gardner 1995). To date, allogeneic skin grafts serve mainly as temporary biological wound dressings that provide a suitable environment for the survival and expansion of autologous small patch or micro skin grafts (Kreis et al. 1989). In the clinic, autologous skin transplantation is the only viable means of achieving permanent closure of massive wounds and the restoration of skin function.

Although great achievements have been made in the understanding of immune responses to allogeneic and xenogeneic skin grafts, the induction of long-term tolerance to of skin grafts is still a grand challenge for immunologists. In this review, we introduce the latest perspectives on skin transplantation immunology and investigate the potential new technologies and methods that offer the prospects of donor-specific tolerance induction to skin grafts.

\section{The Characteristics of Skin Graft Immunology}

Due to the unique tissue structure and cell constitution of the skin, allogeneic skin transplantation leads to different immune responses than solid organ transplantation. 
The rejection of allogeneic skin grafts is considered primarily to be a model of acute graft rejection. As the skin is not a primary vascularised graft, hyperacute rejection, which is a rapidly occurring reaction induced by preexisting alloreactive antibodies in blood, rarely occurs (Colletti et al. 1994). Once placed on the wound, the skin grafts must establish their own blood vascularisation. The skin grafts initially survive through the use of oxygen that is present in the wound bed. The primary trauma and tissue injury result in an inflammatory process with the infiltration of inflammatory cells and accumulation of angiogenic factors around the graft. Over the course of several days, angiogenesis will lead to the ingrowth of vessels (Dresel et al. 2002).

Following the vascularisation of the graft, antigen-presenting cells (APCs) from the donor can migrate out of the skin graft through the lymphatic vessels and infiltrate the draining lymph nodes of the recipient. These cells can present donor antigens directly to the host alloreactive $\mathrm{T}$ cells (i.e., direct antigen-presentation) or through the host APCs (i.e., indirect antigen-presentation), both of which can lead to the activation of host $\mathrm{T}$ cells (Benichou et al. 2011) (Fig. 1a).

Once activated, cytotoxic $\mathrm{CD} 8^{+} \mathrm{T}$ lymphocytes (CTL) and $\mathrm{CD}^{+} \mathrm{T}$ helper (Th) cells become effector cell if specifically recognised alloantigens are encountered. CTL activity, mediated by the perforin-granzyme and Fas-Fas ligand pathways, induces the antigen-specific lysis and apoptosis of donor alloantigen-expressing target cells in the graft, leading to an acute rejection of the graft. Th cells aid the activation and function of $\mathrm{CD}^{+} \mathrm{T}$ cells, which, in turn, cause chronic transplant rejection (Benichou et al. 2011; Binet and Wood 2003).

Although the rejection of allogeneic skin transplants is principally a $\mathrm{T}$ cell-dependent process, inflammation mediated by the innate immune network also contributes to graft-specific immune responses. Transient inflammation at the early stages of transplantation has a marked effect on allograft rejection that is associated with the infiltration of natural killer (NK) cells (Habiro et al. 2005). Conversely, the alloimmune response enhances inflammation injuries. Sensitisation by alloantigens can facilitate innate tissue inflammation and organ injury via a non-specific CD154dependent mechanism (Shen et al. 2010). In alloresponses, keratinocytes may be activated and recruited as non-professional APCs, leading to an amplification of the inflammatory response that may be associated with the susceptibility of skin to immune rejection (Binet and Wood 2003; Haw 1995).

Another important immune cell type involved in the rejection or tolerance of skin grafts is the NK cell. In the conventional theory, NK cells participate in transplantation rejection as effector cells, which can mediate cytolytic effects against allogeneic and xenogeneic cells via the antibody-dependent cell-mediated cytotoxicity mechanism (Kumagai-Braesch et al. 1998) and produce a variety of proinflammatory cytokines that promote the maturation of APCs and indirectly amplify $\mathrm{T}$ cell activation (Kroemer et al. 2008a). Kroemer et al. (2008b) reported that NK cells could reject skin allografts when stimulated with interleukin (IL)-15 in the absence of adaptive immune cells. However, recent studies have demonstrated that NK cells can kill allogeneic graft-derived APCs and prolong the survival of skin grafts through the inhibition of direct $\mathrm{T}$ cell priming or the elimination of activated alloreactive $\mathrm{T}$ cells in a perforin-dependent mechanism (Beilke et al. 2005; Yu et al. 2006). Thus, it is possible that NK cells have bidirectional effects on skin graft rejection and tolerance.

\section{The Immune Effects of Dendritic Cells in Skin Grafts}

In the immune rejection of skin grafts, the Langerhan's cells (LCs) are important APCs that can trigger a $\mathrm{T}$ cell response. LCs are a special and major subpopulation of dendritic cells (DCs) that reside in the epidermis and are most prominent in the stratum spinosum regions. LCs are distinguished from other DCs by the expression of the haematopoietic marker CD45 (LY5), the non-MHC class I molecule CD1a, the intercellular adhesion molecule E-cadherin (CDH1), and the novel C-type lectin Langerin (Merad et al. 2008). Along with this unique phenotype, LCs are estimated to have much longer lifespans and stronger proliferative capacities compared with other DCs (Hemmerling et al. 2011; Vishwanath et al. 2006). These features empower LCs with a potent capacity for antigen presentation and a strong immunogenicity in allotransplantation. Further research confirmed that the immunogenicity of skin grafts is correlated with the distribution and density of LCs (Chen and Silvers 1983). Therefore, it has been widely accepted that donor-derived LCs play an important role in immune responses to allogeneic skin grafts.

Although LCs are the most numerous DC subtype in the skin, other donor-derived APCs in the graft, including dermal DCs and macrophages, can also participate in graft rejection (Benichou et al. 2011; Morelli et al. 2005). The encounters of these APCs with alloreactive recipient $\mathrm{T}$ cells result in direct alloantigen recognition, which can mediate acute immune responses to allogeneic skin grafts (Kazansky 2008).

Alloantigen recognition and graft-specific $\mathrm{T}$ cell activation occur by three mechanisms: the direct, the indirect and the semi-direct pathways (Jiang et al. 2004). Although the indirect pathway is the most common manner by which $\mathrm{T}$ cells detect antigens and is sufficient to induce graft 
rejection, the direct pathway plays an important role in immune responses to skin grafts. In the direct recognition pathway, donor-derived APCs present intact donor MHC molecules but not peptide fragments to host $\mathrm{T}$ cells and induce a potent polyclonal $\mathrm{T}$ cell response in which up to $10 \%$ of the entire peripheral $\mathrm{T}$ cell repertoire is activated, leading ultimately to the rejection of MHC-mismatched grafts (Benichou et al. 2011). Thus, the epidermal LCs and dermal DCs, as the major donor-derived APCs in skin graft, have been shown to be crucial for acute immune responses in skin transplants.

In addition to the vigorous ability to activate $\mathrm{T}$ cell responses, in transplantation, the donor LCs can also induce a special immune response that is independent of costimulatory signalling and the nuclear factor (NF)- $\kappa B$ pathway. The full activation of naïve $\mathrm{T}$ cells requires stimulation through both the $\mathrm{T}$ cell receptor (TCR) and the costimulatory molecule CD28. These events trigger the activation of several intracellular signalling pathways that lead to $\mathrm{T}$ cell proliferation and clonal expansion, cytokine secretion, cell survival, differentiation, and memory cell generation. Signalling through the canonical NF- $\mathrm{BB}$ pathway is critical for proper $\mathrm{T}$ cell activation. In certain cases, inhibition of either the costimulatory signal or the NF- $\mathrm{KB}$ pathway could successfully abrogate the acute allograft rejection process (Finn et al. 2001).

However, a recent study showed that LCs can induce T cells to reach an activation threshold that is sufficient for transplant rejection in the absence of a costimulatory signal or under the condition of impaired NF- $\mathrm{KB}$ pathway, which indicates that LCs can prime $\mathrm{T}$ cells through a bypass signal pathway (Molinero et al. 2008; Rulifson et al. 2002). This finding may explain why certain immune inhibitors, such as rapamycin, which target NF- $\kappa \mathrm{B}$, do not display the expected effects in skin transplantation models that have abundances of LCs (Sun et al. 2008).

Although donor-derived LC-mediated antigen recognition and rejection have been widely accepted, LCs may also participate in transplantation tolerance. In a recent study, Obhrai et al. (2008) showed that in a transgenic LCablation skin transplantation mouse model, the complete absence of LCs could abrogate the long-term survival of minor antigen-mismatched skin grafts, which indicates the possibility that donor LCs are essential for the maintenance of graft tolerance. Thus, the exact contribution of LCs to immune reactions against skin grafts is still a matter of discussion.

\section{Skin Graft Pre-Treatment}

Currently available systemic immunosuppressive agents cannot effectively prevent the rejection of skin grafts.
Moreover, because the majority of skin graft recipients have defects in skin barrier function, immunosuppressive therapy can significantly increase the risk of infection. Therefore, pre-operative treatment of skin allografts to decrease immunogenicity is considered to be a putative solution to improve skin graft survival or even induce longterm tolerance.

\section{Depletion of Donor-Derived DCs}

Because of the crucial role of donor-derived DCs in the rejection of skin grafts, it was hypothesised that the depletion of these cells in grafts or a blockade of the direct antigen recognition process may improve the survival of skin grafts.

9,10-Dimethyl-1,2-benzanthracene (DMBA) was the first chemical agent observed to effectively deplete LCs from the epidermis, as determined by ultrastructural examination (Halliday and Muller 1986). When transplanted onto MHCmismatched recipients, DMBA-treated mouse skin grafts had a prolonged survival time compared to control skin grafts (Odling et al. 1987b). However, because DMBA is a potent chemical carcinogen, the application of DMBA-treated skin grafts to human beings is impractical.

Other than chemical agents, short wavelength ultraviolet light (UV) irradiation can successfully eliminate LCs in skin grafts. Odling et al. (1987a) demonstrated that UVB irradiated skin grafts were depleted of LCs and had significantly longer survival times. We have also used UVC as a tentative pre-treatment for cadaver grafts. In this study, UVC pre-treatment significantly lowered the numbers of APCs in skin samples and thus induced longer survival times of alloskin grafts on patients with full skin thickness burn wounds when combined with an anti- $\beta 2$ microglobulin monoclonal antibody $(\beta 2 \mathrm{mAb})$ preincubation $(\mathrm{Wu}$ et al. 1996). The mean survival time of skin graft survival was $37 \pm 8$ days in the UVC with anti- $\beta 2 \mathrm{mAb}$ group, which was approximately $50 \%$ greater than that observed for the group that was pre-treated with anti- $\beta 2 \mathrm{mAb}$ alone.

However, the LC-free cultured allogeneic epidermal sheets showed only a slightly prolonged survival time (4-5 days) when used as a wound coverage in humans (Aubock et al. 1988), which raises doubts that the depletion of LCs in skin grafts might be clinically viable.

\section{Inhibition of Donor DC Antigen Presentation}

As increasing numbers of research studies indicated that donor-derived DCs contribute to the induction and maintenance of allograft tolerance (Jung et al. 2011; Obhrai et al. 2008), blocking the antigen presentation process in DCs was considered to be a possible and potentially effective therapy for the induction of skin graft tolerance. 
McMinn et al. (1990) reported that gliotoxin, a member of the epipolythiodoxopiperazine family of fungal metabolites, could significantly reduce epidermal LC densities and alter the morphology and function of LCs. Skin grafts that were pre-treated with gliotoxin showed prolonged survival times on MHC-mismatched recipients. When placed onto haplotype-mismatched recipients, gliotoxin pre-treated skin grafts were accepted permanently and were able to induce donor-specific tolerance (McMinn et al. 1990). Other research demonstrated that gliotoxin pre-treatment could prolong the survival of thyroid grafts after transplantation into allogeneic recipients (Sutton et al. 1995). The successes of these grafts demonstrate the potential for gliotoxin as an immunomodulatory agent in organ transplantation. However, a recent study indicated that gliotoxin could cause cell necrosis and apoptosis, especially of Kupffer cells (Anselmi et al. 2007), which raises concern about risks associated with gliotoxin pre-treatment.

Norcantharidin (NCTD), the demethylated form of cantharidin, is a water-soluble synthetic small molecule with immunomodulatory functions, especially of cell lineages that originate from the bone marrow, such as DCs (Chen et al. 2008). NCTD modulates the activity of DCs and inhibits the expression of CD1a, DC-SIGN, and CD83 in a dose-dependent manner. NCTD-treated DCs also stimulate the proliferation of allogeneic $\mathrm{CD}^{+}{ }^{+} \mathrm{Foxp}^{+}$ regulatory $\mathrm{T}$ cells, which might contribute to the induction of donor-specific tolerance. NCTD treatment also resulted in significantly prolonged skin allograft survival in a mouse model (18.2 \pm 2.3 days) in comparison to the non-treated group (11.6 \pm 0.9 days) (Hsieh et al. 2011).

Although the above-mentioned agents appear to be good prospects in the promotion of skin engraftment, the toxicities of these agents might restrict their clinical applications to a certain extent. Photodynamic therapy (PDT) promises to be a novel therapeutic procedure for immune regulation without obvious adverse effects (Mroz and Hamblin 2011). PDT significantly downregulated the expression of $\mathrm{MHC}$ and the costimulatory molecule B7 (60-90\% reduction) on LCs, and this treatment effectively suppressed the ability of LCs to stimulate alloreactive $\mathrm{T}$ cells to proliferate. An experiment showed that the pre-treatment of skin grafts with lowdose PDT could significantly prolong the survival of allografts in a mouse model, from $9.3 \pm 2.2$ to $16.9 \pm 1.7$ days (Obochi et al. 1997). Furthermore, an upregulation of IL-10 protein expression (up to 2.85-fold) was observed in PDTtreated keratocytes in vitro (Byun et al. 2009), which may also contribute to the protective effects in skin allografts.

\section{Prevention of Oxidative Damage}

During allograft rejection, graft-infiltrating inflammatory cells produce high levels of reactive oxygen species (ROS).
These potent oxidants can damage cell membranes and proteins, which are involved in the rejection of allogeneic grafts. Antioxidant therapy has been documented to suppress allograft rejection through the prevention of inflammation and ischemic reperfusion injuries (Ma et al. 2008).

As the skin is a non-primary vascularised graft, more time is required to establish blood flow. The ischemiareperfusion process produces an abundance of ROS, which aggravates damage to the grafts (Baines and Shenkin 2002). A synthetic superoxide dismutase and a catalase mimetic have been proven to protect grafts from ROS injury (Gianello et al. 1996). Tocco et al. used complexes of salen manganese (Salen-Mn), a metal scavenger of ROS that exhibits superoxide dismutase and catalase activities, to inhibit the rejection of fully MHC-mismatched allogeneic skin grafts in mice. The pre-treatment of C57BL/6 donor skin with Salen-Mn complexes strongly impaired the CTL-mediated alloresponse and significantly delayed allograft rejection (Tocco et al. 2006), which indicates that ROS scavengers are promising candidates for the improvement of skin graft survival.

\section{Modification of Xenogeneic Skin Grafts}

It is well-known that allogeneic skin transplantation is an effective protective dressing for patients with massive skin defects; however, the gap between the increasing demand for graft tissues and the limited sources necessitates the discovery of a substitute for cadaver skin. Xenogeneic skin grafts have advantages in both biological activity and availability compared with artificial skin or tissue-engineered autologous skin. Among the mammals, porcine skin has a strong resemblance to human skin with regards to the dermal tissue structure, collagen arrangement, and follicle density (MacLeod et al. 2004). Research has also shown that porcine split-skin grafts are easily prepared, stored and preserved with regard to biological activities (Chiarini et al. 2007). Therefore, porcine skin has been considered as a possible substitute for cadaver skin. However, despite similarities between allogeneic and xenogeneic skin grafts, immune rejection is still a huge obstacle to the use of porcine skin grafts in the long-term coverage or replacement of skin.

At one time, the hyperacute rejection that was mediated by a natural antibody reaction to the Gal $\alpha 1$, 3Gal oligosaccharide on porcine endothelial cells was commonly described as the greatest barrier to the induction of immune tolerance to porcine xenografts (Vaughan et al. 1994). However, unlike vascularised solid organ grafts, the remaining vessels in partial-thickness skin grafts are not often anastomosed with the recipient vessels. A further 
study also confirmed that xenogeneic skin grafts did not elicit stronger Gal-mediated rejection when compared to allogeneic grafts (Gock et al. 2004). Therefore, the rejection of porcine skin grafts is a predominantly cell-mediated response. Based on this premise, many bioengineering or genetic modification strategies have been developed to reduce the vulnerability of porcine grafts to acute rejection (Fig. 1b-g).

\section{Bioengineering Modifications of Skin Graft}

Skin grafts are isolated from vascular circulation when first implanted with a corresponding delay in revascularisation. This delay was traditionally thought to result in ischemic injuries to skin grafts. However, the delay may provide a window of opportunity in which to block the encounters between immune cells and graft antigens or to interrupt the immune effector cell cascade through bioengineering modifications to the grafts.

In recent years, an acellular matrix membrane has been widely studied as a bioengineering modification technique for the improvement of allogeneic and xenogeneic graft acceptance. These acellular membranes, which are mainly composed of an array of laminin, vitrogen, fibronectin, and type IV collagen, can provide an apical surface that remains non-thrombogenic and non-immunogenic. This network of molecules, which comprises the membranes, functions as a barrier to antigen recognition and immune cell migration while simultaneously permitting the free diffusion of nutrients and oxygen (Stubenitsky et al. 2009).

As the vascular endothelial cells are major targets for immune responses, this novel technique was first applied to the vasculature of solid grafts, such as kidney grafts. Pretreatment of a kidney graft with a nano-membrane provided ubiquitous coverage of approximately $90 \%$ of the vascular luminal surface, including both small and large blood vessels, which significantly delayed the onset of rejection without apparent adverse effects on renal function (Brasile et al. 2010).

When used as an artificial interface between skin allografts and wound surfaces, nano-membranes also significantly prolonged graft survival times $(28 \pm 3.8$ vs. $6.8 \pm 1.5$ days $)$ without immunosuppression. Recent studies have implied that these nano-membranes might also serve as drug delivery systems to further improve graft outcomes (Brasile et al. 2011; Stubenitsky et al. 2009). These results demonstrated that the nano-membrane might be a promising method with which we can protect skin grafts from acute rejection.

\section{Genetic Modification of Skin Grafts}

Gene transfer can provide cells with an expressible cloned DNA sequence that encodes a functional protein, with the intent to achieve a therapeutic effect. In gene transfer therapy for the induction of transplantation tolerance, two strategies have been pursued: modification of the graft or modification of the recipient.

Due to the risk of complications associated with the use of viral vectors, the use of gene therapy for patients in the clinic is still years away. Thus, the modification of candidate genes that alter the immune functions of the host, including BAFF, PDL1 and Foxp3, are not viable, although these genes have been proven effective in the improvement of graft tolerance (Ghazizadeh et al. 2012; Walters et al. 2009). To the contrary, it is more practical to construct transgenic animals for use as xenograft donors; therefore, genes and molecules that can ameliorate insults to graft tissues have been studied widely as targets for genetic modification.

Antigen presentation by donor DCs has been welldemonstrated as sufficient to initiate the acute rejection of skin grafts. Thus, the transduction of immunomodulatory genes that can inhibit the function of donor DCs might improve tolerance to skin grafts.

CD200, a membrane glycoprotein that is expressed mainly on the surface of DCs, can transmit an inhibitory signal to certain subsets of $\mathrm{T}$ cells that express CD200R. The CD200/CD200R interaction can suppress the phosphorylation of the ERK1/2 mitogen-activated protein kinase in responding $\mathrm{T}$ cells and contribute to the production of tolerogenic T cells (Gorczynski 2005). Transgenic overexpression of CD200 significantly attenuated the rejection of allografts through the suppression of inflammation and the upregulation of genes that encode Foxp3, transforming growth factor $\beta$, IL-10, and PD-1/PD-L1/PD-L2 in skin grafts, as well as an increased expression of mRNAs for indoleamine 2,3-dioxygenase ( $Y u$ and Gorczynski 2012). However, in this study, both donors and recipients were transfected with CD200; thus, the effect of CD200 overexpression in the graft alone requires further evaluation.

Suppressors of cytokine signalling (SOCS) proteins play pivotal roles in the regulation of macrophage, $\mathrm{T}$ cell, and DC activation, development, and differentiation. Silencing of the SOCS1 gene in DCs through RNA interference can contribute to DC maturity and thus increase immune function. $\mathrm{Fu}$ et al. (2009) reported that SOCS1 genetransduced DCs had lower expression levels of CD40 and CD80 and secreted high levels of IL-10. An infusion of these transgenic DCs prolonged the survival of cardiac allografts, which correlated with a substantial increase in the generation of regulatory $\mathrm{T}$ cells (Fu et al. 2009). These results suggest that SOCS-1 gene transfer may also improve skin graft survival through the inhibition of donorderived DC function.

Full $\mathrm{T}$ cell activation requires a costimulatory signal in addition to the engagement of the TCR with the antigenMHC complexes on APCs. The lack of a costimulatory 


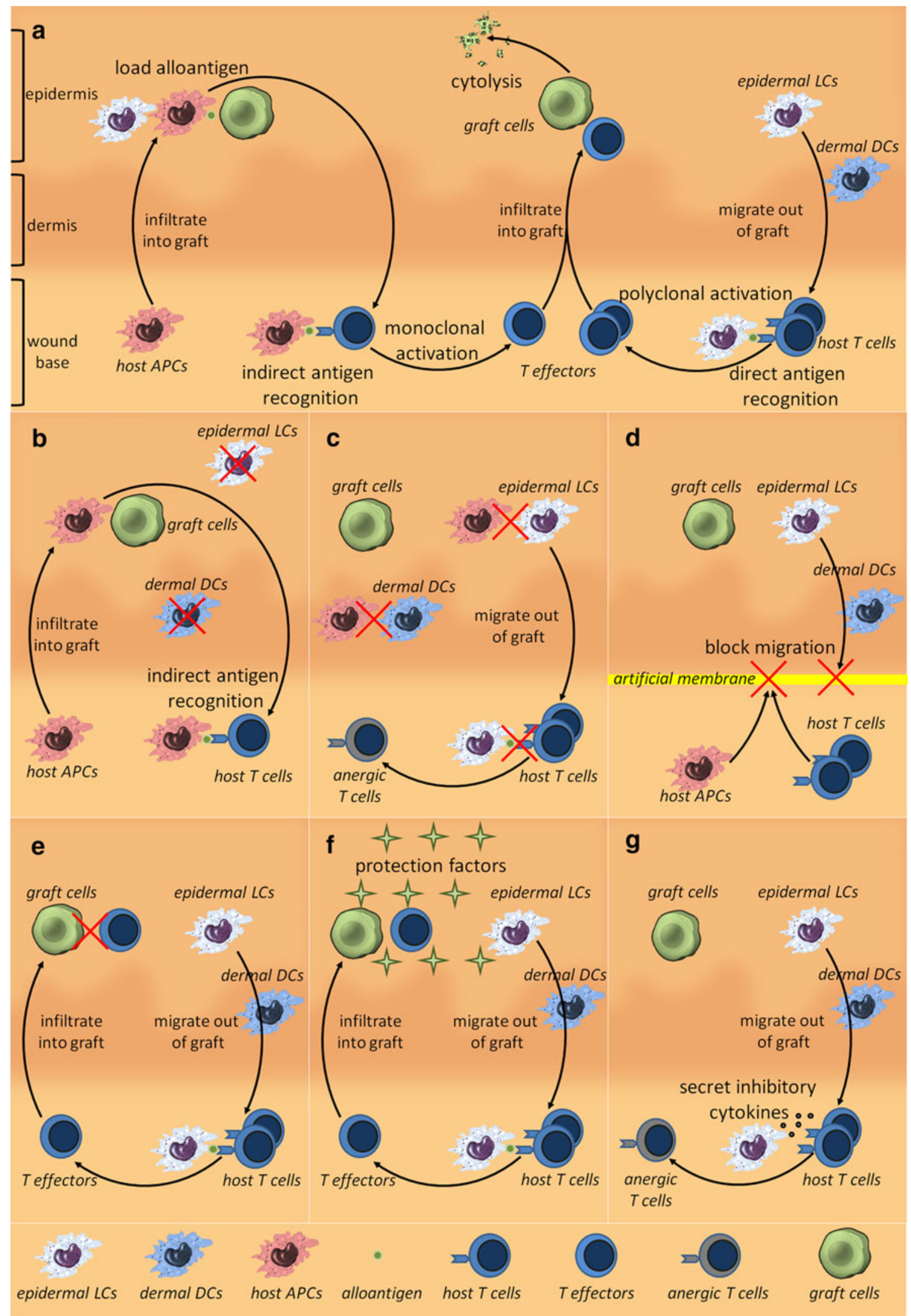


4 Fig. 1 Immune responses to skin grafts and putative strategies for tolerance induction. a Immune responses to skin grafts. b Depletion of donor-derived APCs in the skin graft to block the direct antigen recognition pathway. c Inhibition of the antigen-presenting function of donor-derived APCs or modification of the balance of costimulatory signals to intercept the normal activation process in the host's alloreactive $\mathrm{T}$ cells. d Blocking of the interactions between host immune cells and graft antigens with an artificial matrix membrane. e Importation of protective factors into skin grafts through engineering or genetic modifications to reduce damage to graft cells. f Inhibition of host $\mathrm{T}$ cell function at the effector stage. $\mathbf{g}$ Upregulation of inhibitory cytokine expression by genetic modification

signal after the engagement of the TCR with cognate antigen results in a partial activation of $\mathrm{T}$ cells, which are rendered unresponsive to the encountered antigen, which is a state known as anergy (Durrbach et al. 2010).

Cytotoxic T lymphocyte-associated antigen 4 (CTLA4, $\mathrm{CD} 152)$ is the most important negative costimulatory signal and acts competitively against CD28. CTLA4immunoglobulin (CTLA4-Ig) is a soluble chimeric protein that consists of the extracellular domain of CTLA4 at the $\mathrm{N}$-terminus and the constant and hinge regions of IgG at the C-terminus. With the extracellular CTLA4 domain, CTLA4-Ig binds B7 in the same manner as full-length CTLA4 and functions as a competitive inhibitor of the B7 or CD28 costimulatory pathways.

The soluble CTLA4-Ig Belatacept (trade name Nulojix) has proved to be an effective immunosuppression agent and has been approved for maintenance therapy after renal transplantation. However, the systemic administration of CTLA4-Ig might lead to an increased risk of viral infections or lymphoproliferative disorders (Larsen et al. 2010). Our group has successfully induced a skin-specific overexpression of human CTLA4-Ig in transgenic murine and porcine models (Luo et al. 2012; Wang et al. 2006). The transgenically expressed CTLA4-Ig protein suppressed lymphocyte proliferation in vitro. The survival of transgenic mouse skin grafts on rat wounds was prolonged remarkably compared to wild-type skin grafts from the same mouse strain $(13.7 \pm 4.8$ vs. $7.2 \pm 1.8$ days; $p<0.01)$. Transient levels of circulating CTLA4-Ig protein were detected in recipient sera; however, no extensive immunosuppression was observed (Wang et al. 2008).

When the CTLA4-Ig transgenic porcine skin grafts were applied to patients with partial-thickness burn wounds, the transgenic skin grafts also showed prolonged survival times of approximately 5 days compared with grafts transfected with an empty vector. In a multi-centre, randomised parallel control clinical trial with 200 patients, we observed no detectable systemic side effects that were associated with CTLA4-Ig-gene transfected grafts (Luo et al. 2012).

OX40 (CD134) is a tumor necrosis factor (TNF) receptor related costimulatory molecule that strongly regulates the division and survival of conventional $\mathrm{CD} 4^{+}$ and $\mathrm{CD} 8^{+} \mathrm{T}$ cells and the cross-talk of these cells with APCs (Croft 2010). In a recent study, the ex vivo transfection of allogeneic skin flaps with an OX40immunoglobulin (OX40-Ig) expressing lentiviral vector led to a significantly increased survival time that was correlated with the diminished secretion of IL-2 and interferon (IFN)- $\gamma$. When OX40-Ig was transfected in combination with CTLA4-Ig, the protective effects on the allografts were further enhanced (Zhang et al. 2012).

Cytokines are other important factors that affect transplantation immune responses. The balance among the immunoregulatory cytokines can determine the outcome of the $\mathrm{T}$ cell response. The overexpression of cytokines with negative immunoregulatory functions is assumed to improve graft survival. The local overexpression of IL-10 in donor hearts by an ex vivo intracoronary infusion of a liposome-mediated vector significantly decreased both $\mathrm{CD}^{+}$and $\mathrm{CD}^{+}{ }^{+} \mathrm{T}$ cell responses and expression levels of Th1 cytokine genes such as IL- 2 , IFN- $\gamma$, and TNF- $\alpha$, which led to the prolonged survival of allogeneic hearts in animals (Furukawa et al. 2005; Hong et al. 2002).

Although IL-10 transduction significantly improved the survival of some solid organ grafts and studies showed that IL-10-transduced foetal liver cells could also prolong the survival of mouse skin grafts (Hase et al. 2005), the effects of local IL-10 overexpression on skin grafts, which are more difficult immune rejection models, are still not confirmed.

\section{Conclusions and Future Directions}

In summary, the transplantation of allogeneic and xenogeneic skin grafts is associated with potent immune responses that involve the migration of donor-derived DCs, antigen recognition, activation of host $\mathrm{T}$ cells, and ultimately, the elimination of donor cells and the destruction of grafts. Nevertheless, the immune system is a complicated network. In this process, immune cells, cytokines and signal pathways interact with one another and together lead to graft rejection. The long-term survival of a skin graft requires the delicate control of immune homeostasis to direct the outcome from rejection to donor-specific tolerance.

However, owing to the limited conditions and techniques, current studies normally focus on one factor at a time, and as a consequence, the interactions between these elements have not been thoroughly elucidated. Thus, the results that were obtained in accurately controlled experimental conditions could not always be replicated in other situations or applied in clinical settings. An insight into the interactions between the immune cells that are involved in 
graft rejection and the balance of activating and inhibitory signals in the immune response could help us to find new targets for therapies or effective methods for tolerance induction.

According to current research results, graft pre-treatments or modifications may be promising methods with which to decrease immune responses and improve the survival of skin grafts without general immune suppression. However, in most studies, graft pre-treatments and modifications have exhibited only modest effects. Thus, engineering or genetic modifications may enhance the availability of skin grafts as temporary wound dressings but are not enough to induce long-term donor-specific tolerance. Graft pre-treatments might be combined possibly with sub-therapeutic immunosuppressive drug treatments to augment the effects of pre-treatment and avoid side effects from systemic immunosuppressive therapy.

Acknowledgments This work was supported by Chinese NSFC Grants $(81027004,30571922)$ to J. Wu and G. Luo, “863” grants (2012AA020504) to J. Wu, and State Key Laboratory Funding (SKLZZ200808, SKLZZ200820) to J. Wu and G. Luo.

\section{References}

Anselmi K, Stolz DB, Nalesnik M et al (2007) Gliotoxin causes apoptosis and necrosis of rat Kupffer cells in vitro and in vivo in the absence of oxidative stress: exacerbation by caspase and serine protease inhibition. J Hepatol 47:103-113

Aubock J, Irschick E, Romani N et al (1988) Rejection, after a slightly prolonged survival time, of Langerhans cell-free allogeneic cultured epidermis used for wound coverage in humans. Transplantation 45:730-737

Baines M, Shenkin A (2002) Use of antioxidants in surgery: a measure to reduce postoperative complications. Curr Opin Clin Nutr Metab Care 5:665-670

Beilke JN, Kuhl NR, Van Kaer L et al (2005) NK cells promote islet allograft tolerance via a perforin-dependent mechanism. Nat Med 11:1059-1065

Benichou G, Yamada Y, Yun SH et al (2011) Immune recognition and rejection of allogeneic skin grafts. Immunotherapy 3:757-770

Binet I, Wood KJ (2003) In vivo models of inflammation: immune rejection and skin transplantation in vivo. Methods Mol Biol 225:239-248

Brasile L, Glowacki P, Castracane J et al (2010) Pretransplant kidneyspecific treatment to eliminate the need for systemic immunosuppression. Transplantation 90:1294-1298

Brasile L, Glowacki P, Stubenitsky BM (2011) Bioengineered skin allografts: a new method to prevent humoral response. ASAIO J 57:239-243

Bravo D, Rigley TH, Gibran N et al (2000) Effect of storage and preservation methods on viability in transplantable human skin allografts. Burns 26:367-378

Byun JY, Choi HY, Myung KB et al (2009) Expression of IL-10, TGF-beta(1) and TNF-alpha in cultured keratinocytes (HaCaT Cells) after IPL treatment or ALA-IPL photodynamic treatment. Ann Dermatol 21:12-17

Chen HD, Silvers WK (1983) Influence of Langerhans cells on the survival of $\mathrm{H}-\mathrm{Y}$ incompatible skin grafts in rats. J Invest Dermatol 81:20-23
Chen YJ, Kuo CD, Tsai YM et al (2008) Norcantharidin induces anoikis through Jun-N-terminal kinase activation in CT26 colorectal cancer cells. Anticancer Drugs 19:55-64

Chiarini A, Dal Pra I, Armato U (2007) In vitro and in vivo characteristics of frozen/thawed neonatal pig split-skin strips: a novel biologically active dressing for areas of severe, acute or chronic skin loss. Int J Mol Med 19:245-255

Colletti LM, Johnson KJ, Kunkel RG et al (1994) Mechanisms of hyperacute rejection in porcine liver transplantation. Antibodymediated endothelial injury. Transplantation 57:1357-1363

Croft M (2010) Control of immunity by the TNFR-related molecule OX40 (CD134). Annu Rev Immunol 28:57-78

Dresel A, Kuhn JA, McCarty TM (2002) Sentinel node biopsy site used as full thickness skin graft donor for cutaneous melanoma. Am J Surg 184:176-178

Durrbach A, Francois H, Jacquet A et al (2010) Co-signals in organ transplantation. Curr Opin Organ Transplant 15:474-480

Finn PW, Stone JR, Boothby MR et al (2001) Inhibition of NFkappaB-dependent $\mathrm{T}$ cell activation abrogates acute allograft rejection. J Immunol 167:5994-6001

Fu H, Song S, Liu F et al (2009) Dendritic cells transduced with SOCS1 gene exhibit regulatory DC properties and prolong allograft survival. Cell Mol Immunol 6:87-95

Furukawa H, Oshima K, Tung T et al (2005) Liposome-mediated combinatorial cytokine gene therapy induces localized synergistic immunosuppression and promotes long-term survival of cardiac allografts. J Immunol 174:6983-6992

Gardner CR (1995) The pharmacology of immunosuppressant drugs in skin transplant rejection in mice and other rodents. Gen Pharmacol 26:245-271

Ghazizadeh S, Huang LT, Zhang W (2012) Distinct strategies are required to suppress antigen-specific responses to genetically modified keratinocytes and fibroblasts. Mol Ther 20:196-203

Gianello P, Saliez A, Bufkens X et al (1996) EUK-134, a synthetic superoxide dismutase and catalase mimetic, protects rat kidneys from ischemia-reperfusion-induced damage. Transplantation 62:1664-1666

Gock H, Murray-Segal L, Salvaris E et al (2004) Allogeneic sensitization is more effective than xenogeneic sensitization in eliciting Gal-mediated skin graft rejection. Transplantation 77:751-753

Gorczynski RM (2005) CD200 and its receptors as targets for immunoregulation. Curr Opin Investig Drugs 6:483-488

Habiro K, Shimmura H, Kobayashi S et al (2005) Effect of inflammation on costimulation blockade-resistant allograft rejection. Am J Transplant 5(4 Pt 1):702-711

Halliday GM, Muller HK (1986) Induction of tolerance via skin depleted of Langerhans cells by a chemical carcinogen. Cell Immunol 99:220-227

Hansbrough JF, Mozingo DW, Kealey GP et al (1997) Clinical trials of a biosynthetic temporary skin replacement, DermagraftTransitional Covering, compared with cryopreserved human cadaver skin for temporary coverage of excised burn wounds. J Burn Care Rehabil 18(1 Pt 1):43-51

Hase T, Chargui J, Inori $F$ et al (2005) Human interleukin-10 transduced fetal liver stem cells prolong survival of mouse skin and heart allografts. Transplant Proc 37:287-288

Haw CR (1995) Immunologic roles of keratinocytes: expression of HLA-DR and ICAM-1 on cultured human keratinocytes and their influences on the alloimmune response. J Dermatol 22:839-844

Hemmerling J, Wegner-Kops J, von Stebut E et al (2011) Human epidermal Langerhans cells replenish skin xenografts and are depleted by alloreactive $\mathrm{T}$ cells in vivo. $\mathrm{J}$ Immunol 187:1142-1149

Hong YS, Laks H, Cui G et al (2002) Localized immunosuppression in the cardiac allograft induced by a new liposome-mediated IL10 gene therapy. J Heart Lung Transplant 21:1188-1200 
Hsieh CH, Liao HF, Kuo CD et al (2011) Norcantharidin modulates development of dendritic cells and prolongs skin allograft survival. Transplantation 92:848-857

Jiang S, Herrera O, Lechler RI (2004) New spectrum of allorecognition pathways: implications for graft rejection and transplantation tolerance. Curr Opin Immunol 16:550-557

Jung KC, Park CG, Jeon YK et al (2011) In situ induction of dendritic cell-based $\mathrm{T}$ cell tolerance in humanized mice and nonhuman primates. J Exp Med 208:2477-2488

Kazansky DB (2008) MHC restriction and allogeneic immune responses. J Immunotoxicol 5:369-384

Kreis RW, Vloemans AF, Hoekstra MJ et al (1989) The use of nonviable glycerol-preserved cadaver skin combined with widely expanded autografts in the treatment of extensive third-degree burns. J Trauma 29:51-54

Kroemer A, Edtinger K, Li XC (2008a) The innate natural killer cells in transplant rejection and tolerance induction. Curr Opin Organ Transplant 13:339-343

Kroemer A, Xiao X, Degauque N et al (2008b) The innate NK cells, allograft rejection, and a key role for IL-15. J Immunol 180:7818-7826

Kumagai-Braesch M, Satake M, Qian Y et al (1998) Human NK cell and ADCC reactivity against xenogeneic porcine target cells including fetal porcine islet cells. Xenotransplantation 5:132-145

Larsen CP, Grinyo J, Medina-Pestana J et al (2010) Belatacept-based regimens versus a cyclosporine A-based regimen in kidney transplant recipients: 2-year results from the BENEFIT and BENEFIT-EXT studies. Transplantation 90:1528-1535

Luo G, Huang Z, Tan J et al (2012) Promoting wound healing in burn patients: pig-derived tissues. Regenerative Medicine in China. Science 336(6080):40-41. doi:10.1126/science.336.6080.497-c

Ma A, Qi S, Chen H (2008) Antioxidant therapy for prevention of inflammation, ischemic reperfusion injuries and allograft rejection. Cardiovasc Hematol Agents Med Chem 6:20-43

MacLeod TM, Sarathchandra P, Williams G et al (2004) Evaluation of a porcine origin acellular dermal matrix and small intestinal submucosa as dermal replacements in preventing secondary skin graft contraction. Burns 30:431-437

McMinn PC, Halliday GM, Muller HK (1990) Effects of gliotoxin on Langerhans' cell function: contact hypersensitivity responses and skin graft survival. Immunology 71:46-51

Merad M, Ginhoux F, Collin M (2008) Origin, homeostasis and function of Langerhans cells and other Langerin-expressing dendritic cells. Nat Rev Immunol 8:935-947

Molinero LL, Zhou P, Wang Y et al (2008) Epidermal Langerhans cells promote skin allograft rejection in mice with NF-kappa B-impaired T cells. Am J Transplant 8:21-31

Morelli AE, Rubin JP, Erdos G et al (2005) CD4 + T cell responses elicited by different subsets of human skin migratory dendritic cells. J Immunol 175:7905-7915

Mroz P, Hamblin MR (2011) The immunosuppressive side of PDT. Photochem Photobiol Sci 10:751-758

Obhrai JS, Oberbarnscheidt M, Zhang N et al (2008) Langerhans cells are not required for efficient skin graft rejection. J Invest Dermatol 128:1950-1955

Obochi MO, Ratkay LG, Levy JG (1997) Prolonged skin allograft survival after photodynamic therapy associated with modification of donor skin antigenicity. Transplantation 63:810-817
Odling KA, Halliday GM, Muller HK (1987a) Effects of low or high doses of short wavelength ultraviolet light (UVB) on Langerhans cells and skin allograft survival. Immunol Cell Biol 65(Pt 4):337-343

Odling KA, Halliday GM, Muller HK (1987b) Enhanced survival of skin grafts depleted of Langerhans' cells by treatment with dimethylbenzanthracene. Immunology 62:379-385

Richters CD, Hoekstra MJ, du Pont JS et al (2005) Immunology of skin transplantation. Clin Dermatol 23:338-342

Rulifson IC, Szot GL, Palmer E et al (2002) Inability to induce tolerance through direct antigen presentation. Am J Transplant 2:510-519

Shen X, Reng F, Gao F et al (2010) Alloimmune activation enhances innate tissue inflammation/injury in a mouse model of liver ischemia/reperfusion injury. Am J Transplant 10:1729-1737

Stubenitsky BM, Brasile L, Rebellato LM et al (2009) Delayed skin allograft rejection following matrix membrane pretreatment. J Plast Reconstr Aesthet Surg 62:520-525

Sun Q, Liu Q, Zheng Y et al (2008) Rapamycin suppresses TLR4triggered IL-6 and PGE(2) production of colon cancer cells by inhibiting TLR4 expression and NF-kappaB activation. Mol Immunol 45:2929-2936

Sutton P, Moreland A, Hutchinson IV et al (1995) Investigation of the potential use of immunosuppressive agent gliotoxin in organ transplantation. Transplantation 60:900-902

Tocco G, Illigens BM, Malfroy B et al (2006) Prolongation of alloskin graft survival by catalytic scavengers of reactive oxygen species. Cell Immunol 241:59-65

Vaughan HA, Loveland BE, Sandrin MS (1994) Gal alpha(1,3)Gal is the major xenoepitope expressed on pig endothelial cells recognized by naturally occurring cytotoxic human antibodies. Transplantation 58:879-882

Vishwanath M, Nishibu A, Saeland S et al (2006) Development of intravital intermittent confocal imaging system for studying Langerhans cell turnover. J Invest Dermatol 126:2452-2457

Walters S, Webster KE, Sutherland A et al (2009) Increased CD4+Foxp3+T cells in BAFF-transgenic mice suppress $\mathrm{T}$ cell effector responses. J Immunol 182:793-801

Wang Y, Ni Y, Wei H et al (2006) Stable skin-specific overexpression of human CTLA4-Ig in transgenic mice through seven generations. Acta Biochim Biophys Sin 38:171-178

Wang Y, Wei H, Ni Y et al (2008) Transgenic expression of cytotoxic T-lymphocyte-associated antigen 4-immunoglobulin prolongs xenogeneic skin graft survival without extensive immunosuppression in rat burn wounds. J Trauma 65:154-162

Wu J, Barisoni D, Armato U (1996) Prolongation of survival of alloskin grafts with no concurrent general suppression of the burned patient's immune system: a preliminary clinical investigation. Burns 22:353-358

Yu K, Gorczynski RM (2012) Persistence of gene expression profile in CD200 transgenic skin allografts is associated with graft survival on retransplantation to normal recipients. Transplantation 94:36-42

$\mathrm{Yu}$ G, Xu X, Vu MD et al (2006) NK cells promote transplant tolerance by killing donor antigen-presenting cells. J Exp Med 203:1851-1858

Zhang J, Miao Q, Yang Y et al (2012) Effect of combined OX40Ig and CTLA4Ig gene local transfer on allograft rejection and the underlying mechanisms. J Surg Res 178:949-958 